\title{
CTHRC1 overexpression promotes cervical carcinoma progression by activating the Wnt/PCP signaling pathway
}

\author{
MEI ZHENG ${ }^{1 *}$, QIN ZHOU ${ }^{2 *}$, XINGGUANG LIU ${ }^{2}$, CHENGZE WANG $^{2}$ and GANGLI LIU ${ }^{2}$ \\ ${ }^{1}$ Department of Traditional Chinese Medicine, Qianfoshan Hospital Affiliated to Shandong University, Jinan, \\ Shandong 250014; ${ }^{2}$ School of Stomatology, Shandong University, Shandong Provincial Key Laboratory of \\ Oral Tissue Regeneration, Jinan, Shandong 250012, P.R. China
}

Received December 21, 2017; Accepted January 7, 2019

DOI: $10.3892 / o r .2019 .6963$

\begin{abstract}
The tumorigenesis and metastasis of tumors are associated with human collagen triple helix repeats containing 1 (CTHRC1). To study the effects and possible impacting mechanisms of CTHRC1 on human cervical carcinoma development, samples of paraffin-embedded cervical carcinoma and HeLa cells were examined. Immunofluorescence, cell wound scratch assay, western blot analysis and Transwell invasion assay were used to evaluate HeLa cells in response to silencing of the CTHRC1 gene in cervical carcinoma. The expression levels of gap-associated proteins of the Wnt/PCP pathway in paraffin-embedded cervical carcinoma samples were also evaluated by immunohistochemical staining. CTHRC1 promoted the migration and invasion of HeLa cells in vitro, downregulated Ror2 and p-c-Jun and activated the Wnt/PCP pathway. Furthermore, the expression of p-c-Jun, Ror2 and Wnt5a was increased after overexpression of CTHRC1 as revealed in HeLa cells compared to control group. The present experiments revealed that CTHRC1 promoted HeLa cell progression by activating the Wnt/PCP signaling pathway and may play a key role in the invasion and metastasis of cervical carcinoma.
\end{abstract}

\section{Introduction}

Human cervical carcinoma is one of the most preventable cancer types in women. Although an effective early screening tool is available for cervical cancer, it remains the third leading cause of female cancer-related deaths with a mortality

Correspondence to: Professor Gangli Liu, School of Stomatology, Shandong University, Shandong Provincial Key Laboratory of Oral Tissue Regeneration, 44-1 West Wenhua Road, Jinan, Shandong 250012, P.R. China

E-mail: liugangli@sdu.edu.cn

*Contributed equally

Key words: collagen triple helix repeat containing 1, Wnt/PCP pathway, HeLa, migration, invasion, cervical carcinoma rate near 30\% within 5 years of diagnosis (1). Therefore, it is critical to explore and understand the mechanisms concerning the development and metastasis of cervical cancer, which may help us impede cervical cancer progression.

CTHRC1 is an extracellular matrix protein that is associated with atherosclerosis (2). Recently, CTHRC1 was found to be upregulated during the tumorigenesis and metastasis of certain cancers (3) and promoted cancer cell invasion and migration (4-6). A recent study suggested that CTHRC1 enhances tumor cell invasion and metastasis by promoting epithelial-mesenchymal transition (EMT), invasion, migration and/or angiogenesis through several signaling pathways (7). Recently, studies have suggested that Cthrcl may activated the non-canonical planar cell polarity (PCP) pathway, one of the 3 Wnt signaling pathways.

Although Cthrcl is upregulated during tumorigenesis and metastasis, the effects and actual mechanisms during those processes have not been thoroughly elucidated to date.

HeLa cells were used in the present study to observe the expression and changes of CTHRC1 in cervical carcinoma and to demonstrate the function of CTHRC1 in the PCP pathway of non-canonical Wnt signaling.

\section{Materials and methods}

Antibodies and reagents. Rabbit phospho-GSK-3 $\beta$ antibodies and monoclonal phospho-Akt (Ser473) were purchased from the Cell Signaling Technologies, Inc. (Beverly, MA, USA; cat. nos. 5558 and 4060, respectively). Rabbit monoclonal CTHRC1, C-myc and Ror2 antibodies (tyrosine-protein kinase transmembrane receptor Ror2) were purchased from Abcam, Inc. (Cambridge, MA, USA; cat. nos. ab85739, ab32072 and ab92379, respectively). Rabbit polyclonal c-Jun and Wnt5a antibodies were obtained from WanleiBio (Shenyang, China; cat. nos. wl0219a and wl0198, respectively). Goat anti-rabbit HRP-conjugated secondary antibodies and goat monoclonal HRP-conjugated antibody against GAPDH were obtained from Dingguo Biological Technology (Beijing, China; cat. nos. IH-0011 and SH-0031, respectively).

Patients and tissue specimens. Cervical carcinoma tissue specimens $(\mathrm{n}=37)$ were collected from patients following radical surgery between January 2005 and December 2015 
at Qianfoshan Hospital Affiliated to Shandong University (Jinan, China) with informed consent obtained concerning the use of surgically resected specimens for research purposes. All human tissue and sample experiments were approved by the Ethics Committee of the School of Stomatology, Shandong University. No patient received any form of adjuvant therapy before surgery.

Cell culture. HeLa cells were cultured in Dulbecco's modified Eagle's medium (DMEM) containing 10\% fetal bovine serum (FBS) and $1 \%$ penicillin-streptomycin. The cells were cultured in a humidified atmosphere of $5 \% \mathrm{CO}_{2}$ and $95 \%$ air at $37^{\circ} \mathrm{C}$.

siRNA synthesis. Single siRNA1 strand was, GGTGGTGGA CCTGTATAAT; single siRNA2 strand was, GCTGTCAGC GTTGGTATTT and single siRNA3 strand was, GGAGAT GCTTCTACTGGAT. All single siRNA strands were synthesized at Invitrogen; Thermo Fisher Scientific, Inc. (Waltham, MA, USA).

siRNA transfection. The plasmid pcDNA3.1-CTHRC1 was constructed by Shinegene (Shanghai, China) and the sequence of human CTHRC1 was obtained from PubMed (NM-138455.3). siRNA (1, 2 and 3) sequences were designed by Guangzhou RiboBio Co., Ltd. (Guangzhou, China). HeLa cells $\left(2 \times 10^{5}\right)$ were seeded in 6 -well culture plates. Plasmids (pcDNA3.1-CTHRC1 and pcDNA3.1) and siRNA were transfected into cells using PolyJet reagent according to the manufacturer's instructions (Invitrogen; Thermo Fisher Scientific, Inc.), when the cultures reached $\sim 80 \%$ confluence. For pcDNA3.1 or pcDNA3.1-CTHRC1 single plasmid transfection, $1 \mu \mathrm{g}$ of DNA, $100 \mu \mathrm{l}$ jetPRIME buffer and $2 \mu \mathrm{l}$ jetPRIME/well were used in a 6-well plate. Briefly, $1 \mu \mathrm{g}$ DNA was diluted into $100 \mu$ l jetPRIME ${ }^{\circledR}$ buffer and mixed by vortexing. Then, $2 \mu \mathrm{l}$ jetPRIME ${ }^{\circledR}$ was added, vortexed and incubated for $10 \mathrm{~min}$ at room temperature (RT). Transfection mix $(100 \mu \mathrm{l}) /$ well was added onto the cells, and distributed evenly by gently rocking the plates back and forth. Medium was replaced after $6 \mathrm{~h}$ of transfection. For plasmid and siRNA co-transfection, $1 \mu \mathrm{g}$ DNA and $20 \mathrm{nM}$ siRNA/well were used in a 6 -well plate with $100 \mu \mathrm{l}$ jetPRIME buffer and $2 \mu \mathrm{l}$ jetPRIME. After $48 \mathrm{~h}$ of incubation, the cells were harvested for further analysis. Efficiency of the transfection was assessed by western blotting.

Wound scratch assay. Using $\alpha$-DMEM as complete cell culture medium, 3-4x $10^{6} \mathrm{HeLa}$ cells were seeded at a high density in 6 -well plates after serum-free incubation for 12 or $24 \mathrm{~h}$ and were allowed to attach to $80 \%$ density. HeLa cell wounds were made by scraping through the cell monolayer with $200-\mu$ l sterile pipette tips. Cells of each well were completely exposed to serum-free $\alpha$-DMEM for up to $12 \mathrm{~h}$ with or without siRNA transfection. Images were acquired at a magnification of x100 under a phase-contrast microscope at different time-points. The widths of the healing area in the cell monolayer were quantified and calculated using Image-Pro Plus 6.0 software (Media Cybernetics, Inc., Rockville, MD, USA).

Transwell invasion assay. The Matrigel-coated filter system was used to evaluate cell invasion. HeLa cells were disaggregated and resuspended in $\alpha$-MEM with $0.2 \%$ FBS, and $2 \times 10^{4}$ cells were placed into the upper chamber of the Matrigel-coated Transwell inserts (Vigorous Biotechnology, Beijing, China) (8- $\mu \mathrm{m}$ pore size) precoated with Matrigel and exposed to serum-free medium with or without siRNA. $\alpha$-MEM supplemented with $10 \%$ FBS was placed in the lower chamber, and cells were incubated for $24 \mathrm{~h}$ at $37^{\circ} \mathrm{C}$ with $95 \%$ air and $5 \% \mathrm{CO}_{2}$ for $24 \mathrm{~h}$. Next, the invaded cells were fixed with $100 \%$ cold methanol for $15 \mathrm{~min}$ and were stained with $0.5 \%$ crystal violet in $0.01 \mathrm{ml}$ of phosphate-buffered saline (PBS) for $10 \mathrm{~min}$, and then the non-invasive cells remaining on the upper membrane were removed with a cotton wool. The number of invaded cells, which penetrated the lower side of the filter, was counted ( 8 fields/filter), and images were captured under a light inverted microscope at a magnification of x200, as aforementioned.

Western blotting. HeLa cells were washed twice with ice-cold PBS, harvested and then lysed in RIPA buffer (Beyotime Institute of Biotechnology, Shanghai, China).

The protein concentration was detected using the BCA protein assay kit. Equal amounts of total protein $(10 \mu \mathrm{g})$ were separated by $10 \%$ SDS-PAGE electrophoresis and were transferred onto polyvinylidene difluoride (PVDF) membranes (EMD Millipore, Bedford, MA, USA). After blocking in 5\% non-fat dry milk in Tween-20/Tris-buffered saline for $1 \mathrm{~h}$, the membranes were probed with various antibodies against Cthric1 (dilution 1:5,000; Abcam, Inc.), Ror2 (dilution 1:2,000), Wnt5a (dilution 1:1,000), p-c-Jun (dilution 1:500) and GAPDH (dilution 1:20,000) overnight at $4^{\circ} \mathrm{C}$, and were subsequently incubated with a species-specific HRP-conjugated secondary antibody (dilution 1:20,000) for $1 \mathrm{~h}$ at $25^{\circ} \mathrm{C}$. Finally, peroxidase activity on the PVDF membranes was assessed on X-ray film using ECL Western Blotting Studio Software (Beyotime Institute of Biotechnology).

Immunohistochemical staining. The expression of Cthric1 in cervical carcinoma specimens was assessed in immunofluorescence studies. Immunohistochemistry was performed using a two-step standard protocol. In simple terms, human cervical carcinoma sample tissues were prepared by $4 \% \mathrm{w} / \mathrm{v}$ paraformaldehyde. The tissues were deparaffinized in xylene, hydrated through a graded alcohol series and washed with PBS as aforementioned. After incubating overnight with Cthric1 at $4^{\circ} \mathrm{C}$ (dilution 1:500) the slides were washed and incubated with HRP-conjugated goat anti-rabbit secondary antibody (dilution 1:1,000) for $30 \mathrm{~min}$ at room temperature. Sections treated with PBS instead of the primary antibody were used as negative controls. To observe Cthricl expression, we used a method described by Maruyama et al (8). The absence of membrane expression and positive cytoplasmic and nuclear expression were considered to indicate abnormal Cthric1 expression. The tissues sections were imaged using a confocal fluorescence microscope.

Immunofluorescence. HeLa cells were grown on glass coverslips in 6-well plates with or without siRNA for 24 or $48 \mathrm{~h}$. HeLa cells were fixed with paraformaldehyde for $15 \mathrm{~min}$, and then were washed with PBS at room temperature. After blocking with normal goat serum, HeLa cells were incubated with the 
A

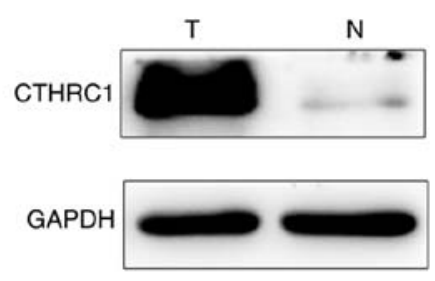

B

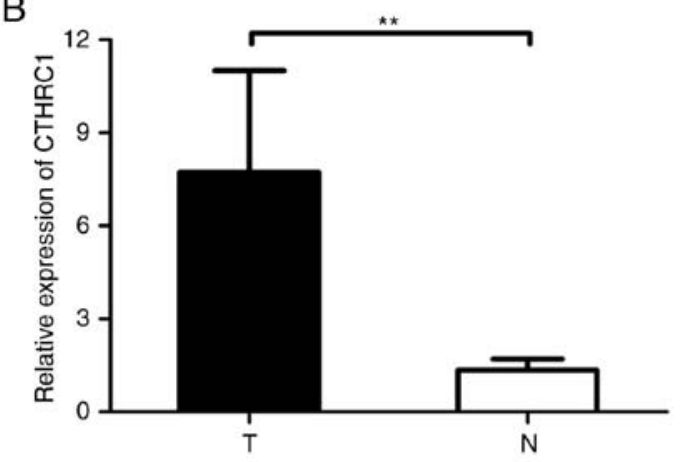

C

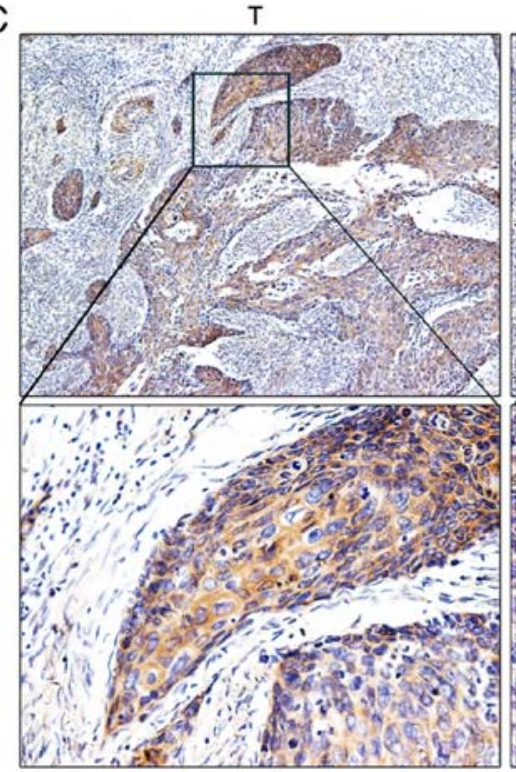

T

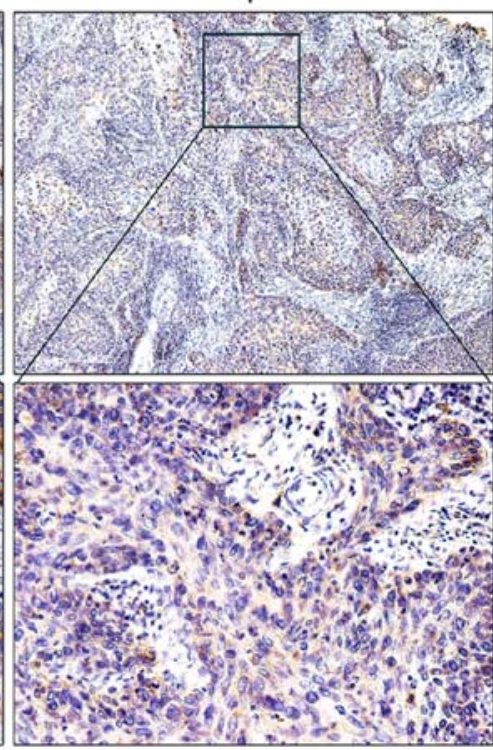

$\mathrm{AE}$

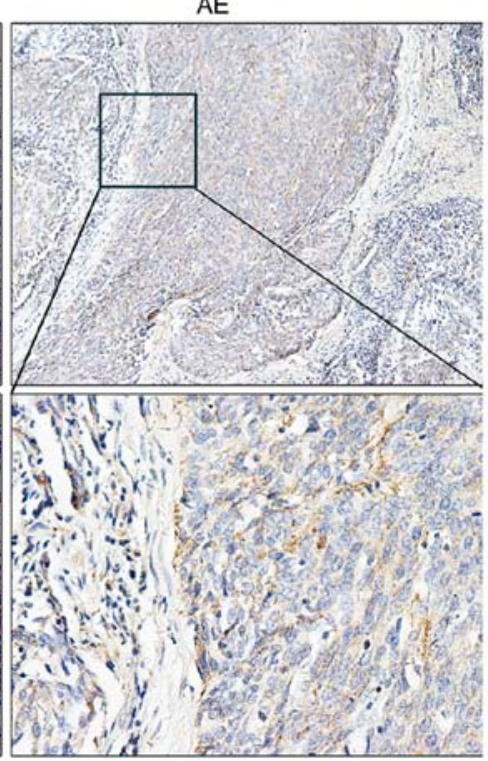

Figure 1. Representative images of immunohistochemical staining of CTHRC1 in cervical cancer tissues. (A and B) CTHRC1 expression in cervical cancer tissues and normal tissues was analyzed by western blotting; ${ }^{* *} \mathrm{P} \leq 0.01$. (C) CTHRC1 was abundant in cervical cancer tissues as revealed by immunohistochemical staining. CTHRC1, collagen triple helix repeat containing 1; T, tumor tissues; AE, adjacent non-cancerous tissues.

associated antibody ( $\beta$-catenin; dilution 1:200; cat. no. AC106) at $4{ }^{\circ} \mathrm{C}$ overnight followed by incubation with FITC-conjugated goat anti-rabbit $\mathrm{IgG}$ (cat. no. IH-0011) at room temperature for $1 \mathrm{~h}$. The slides were washed in PBS-Tween-20 (0.1\%) before being stained with DAPI, and the immunofluorescence signals were visualized and recorded with a fluorescence microscope.

Statistical analysis. Statistical analyses were performed using SPSS 19.0 software (SPSS, Inc., Chicago, IL, USA). Comparison between groups was performed using paired Student's t-test, and for multiple comparisons significant levels were adjusted for the number of tests. The experimental and the control group of cells using independent sample t-test. All of the results were expressed as the means \pm standard deviation (SD). A value of $\mathrm{P}<0.05$ was considered to indicate a statistically significant result. The survival analysis of patients was evaluated using Cox proportional hazards regression analysis and the Kaplan Meier method, and significant differences were examined using the log-rank test.

\section{Results}

CTHRCl expression is increased in cervical carcinoma patients. To reveal whether CTHRC1 is expressed in cervical carcinoma and its effect, we collected 37 paraffin-embedded samples of cervical carcinoma. Western blotting revealed that CTHRC1 expression was significantly upregulated in tumor tissues compared with that in adjacent non-cancerous tissues (Fig. 1A and B). CTHRC1 was further revealed by immunohistochemical analysis of 37 tumor tissue specimens from patients. CTHRC1 expression was found to be higher in cancer tissue compared to adjacent non-cancerous epithelial tissues (Fig. 1C). Additionally, we observed by immunofluorescence that CTHRC1 was abundant in tumor tissues (Fig. 2).

Relationship between survival in cervical carcinoma patients and CTHRC1 expression. To detect the relationship between CTHRC1 expression and cervical carcinoma patients, the correlation between CTHRC1 expression and the survival status of patients was analyzed. It appears that the patients with low expression had better prognosis and patients with high expression had worse prognosis $(\mathrm{P}=0.008$; Fig. 3$)$.

To further evaluate whether CTHRC1 expression represents a prognostic parameter in cervical carcinoma patients, regression analysis using Cox proportional hazards model was performed. In univariate analysis, variables such as low CTHRC1 expression and $\mathrm{pN}^{+}$stage revealed a significant higher hazard ratio (HR) for a significantly better prognosis. 


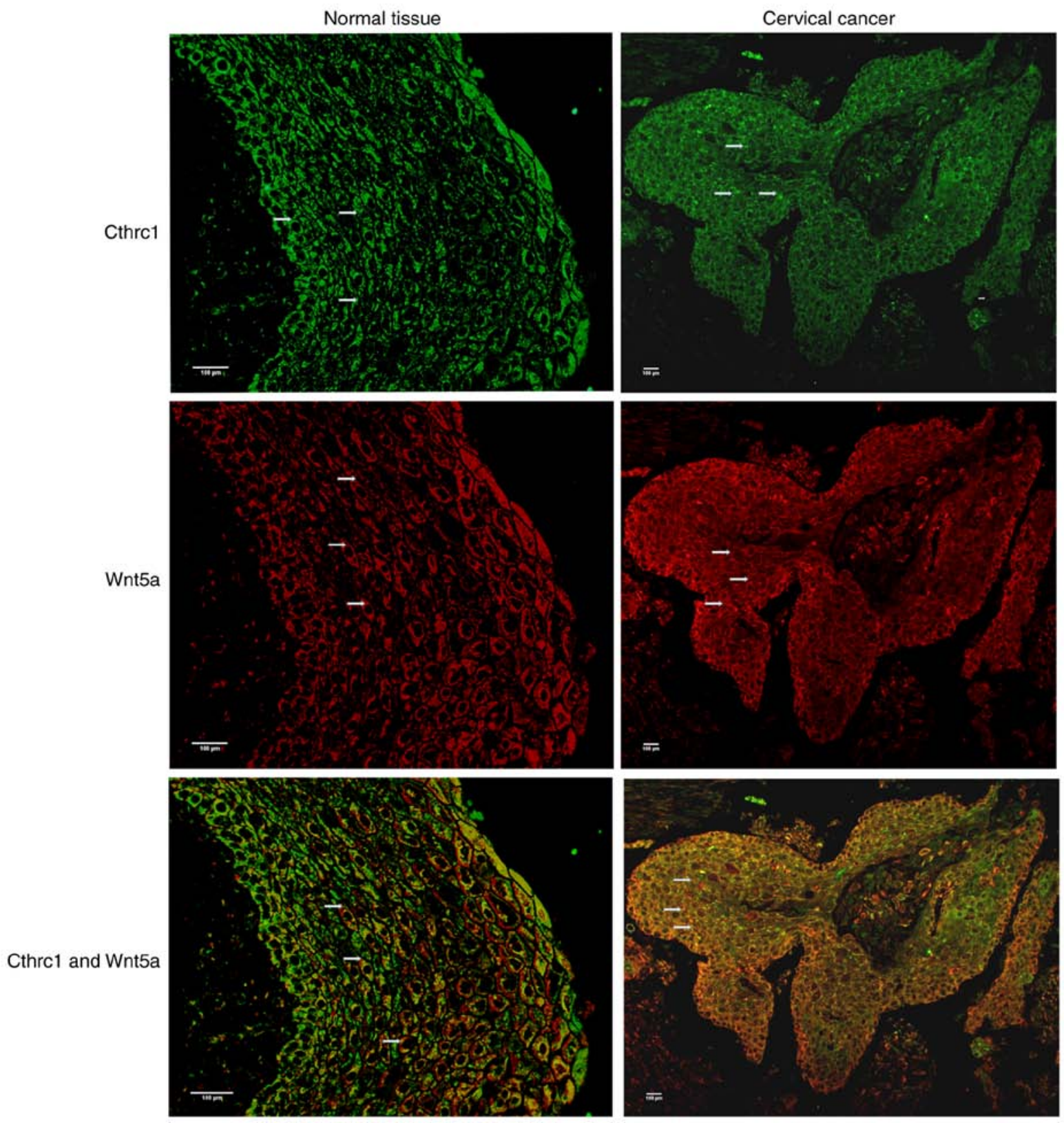

Figure 2. High differentiation of cervical cancer, with increasing signals of CTHRC1 in normal tissue. Immunofluorescence staining of CTHRC1 and Wnt5a in cervical cancer samples, where green color represents CTHRC1 staining of the membrane (first line), red color represents Wnt5a staining of the membrane (second line) and co-localization of CTHRC1 and Wnt5a (third line) (scale bar, $10 \mu \mathrm{m}$ ). The first line, white arrows represent CTHRC1 positive cells. In the second line, white arrows represent Wnt5a positive cells. In the third line, white arrows represent CTHRC1 and Wnt5a positive cells. Immunoreactivity of CTHRC1 was abundant in high differentiation of cervical cancer and there was intensive CTHRC1 immunoreactive signals. CTHRC1, collagen triple helix repeat containing 1 .

Furthermore, multivariate analysis was performed using significant variables surveyed in univariate analysis. The results indicated that CTHRC1 expression was the only independent prognostic predictor $(\mathrm{P}=0.031$; Table I). These results significantly indicated that downregulated CTHRC1 expression in cervical carcinoma patients was strongly associated with a better prognosis.

Knockout of CTHRCl reduces cervical cancer cell invasion and migration in vitro. To explore the effect of CTHRC1 expression, the correlation of CTHRC1 knockout treatment and the biological behaviors of HeLa cells was investigated. CTHRC1 siRNA2 (the most effective siRNA compared to CTHRC1
siRNA1 and CTHRC1 siRNA3) was chosen to knockout CTHRC1 protein (Fig. 4A). When CTHRC1 was knocked out, the expression of Wnt5a, Ror2 and p-c-Jun was downregulated as revealed by western blotting (Fig. 4B). Compared with the control group, the CTHRC1 group exhibited significant decreased migration and invasion abilities as determined by Transwell invasion and wound scratch assays (Fig. 4C and D). As shown in Fig. 4C, the number of HeLa cells that invaded to the lower side of the Transwell membrane in the knockdown CTHRC1 group was significantly lower than that of the control group, indicating that CTHRC1 may promote the invasion of HeLa cells. Fig. 4D revealed that in the wound scratch assay, the control cells appear to show more healing ability compared 


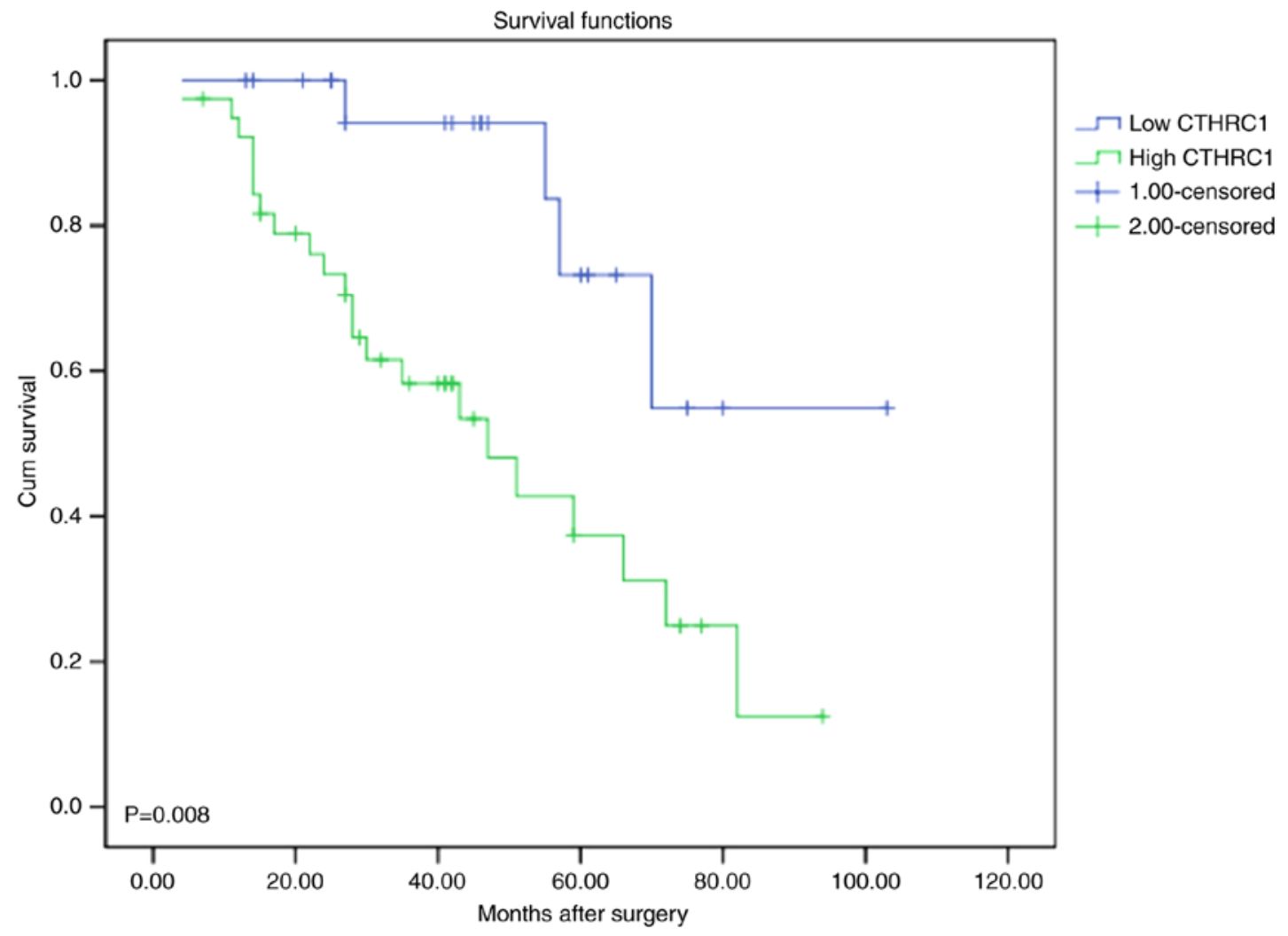

Figure 3. Kaplan-Meier survival analysis for cervical cancer patients. The P-value was determined using the log-rank test. Survival time was calculated based on the date of surgery and the last follow-up. Comparison of the overall survival (OS) between cervical cancer patients with low-CTHRC1 and high-CTHRC1 expression tumors. CTHRC1, collagen triple helix repeat containing 1.

Table I. Cox proportional hazards model analysis of variables affecting survival in cervical carcinoma patients.

\begin{tabular}{|c|c|c|c|c|c|}
\hline \multirow[b]{2}{*}{ Variables } & \multirow[b]{2}{*}{ Categories } & \multicolumn{2}{|c|}{ Univariate analysis } & \multicolumn{2}{|c|}{ Multivariate analysis } \\
\hline & & HR $(95 \% \mathrm{CI})$ & P-value & $\mathrm{HR}(95 \% \mathrm{CI})$ & P-value \\
\hline Age (years) & $<55 / \geq 55$ & $1.384(0.738-3.533)$ & 0.227 & & \\
\hline Differentiation & $\begin{array}{l}\text { Moderately+ } \\
\text { poorly/well }\end{array}$ & $1.249(0.623-2.655)$ & 0.458 & & \\
\hline pT stage & T3-4/T1-2 & $1.635(0.714-3.453)$ & 0.220 & & \\
\hline pTNM stage & III-IV/I-II & $2.029(0.961-4.719)$ & 0.063 & & \\
\hline pN stage & $\mathrm{N}^{+} / \mathrm{N}^{0}$ & $2.219(1.055-5.095)$ & 0.034 & $1.581(0.698-3.623)$ & 0.272 \\
\hline CTHRC1 & High/low & $3.937(1.325-11.562)$ & 0.012 & $3.374(1.201-9.294)$ & 0.031 \\
\hline
\end{tabular}

HR, hazard ratio; CI, confidence interval; pTNM, pathological tumor-node-metastasis stage; CTHRC1, collagen triple helix repeat containing 1. Bold print indicates statistical significance, $\mathrm{P} \leq 0.05$.

to the siRNA group at $12 \mathrm{~h}$, indicating that CTHRC1 knockdown influenced the migration behavior of HeLa cells. The data demonstrated that CTHRC1 could regulate the invasion and migration of HeLa cells in vitro.

Overexpression of CTHRC1 promotes HeLa cell migration and invasion in vitro. CTHRC1 expression was further assessed in HeLa cell lines. Western blotting revealed that higher levels of CTHRC1 protein and mRNA expression were detected in HeLa cells when compared to the vector (Fig. 5A), and then further in vitro study was performed. The relationship between the biological behaviors and CTHRC1 overexpression in HeLa cells was assessed. When CTHRC1 was overexpressed, the expression of p-c-Jun, Ror2 and Wnt5a were increased by western blotting (Fig. 5A). HeLa cell invasion and migration were markedly increased when CTHRC1 was overexpressed using Transwell invasion and wound scratch assays (Fig. 5B-D). As revealed in Fig. 5C and D, in the wound scratch assay, the CTHR1 overexpressed cells appear to show more healing ability compared to the control group at $12 \mathrm{~h}$, 

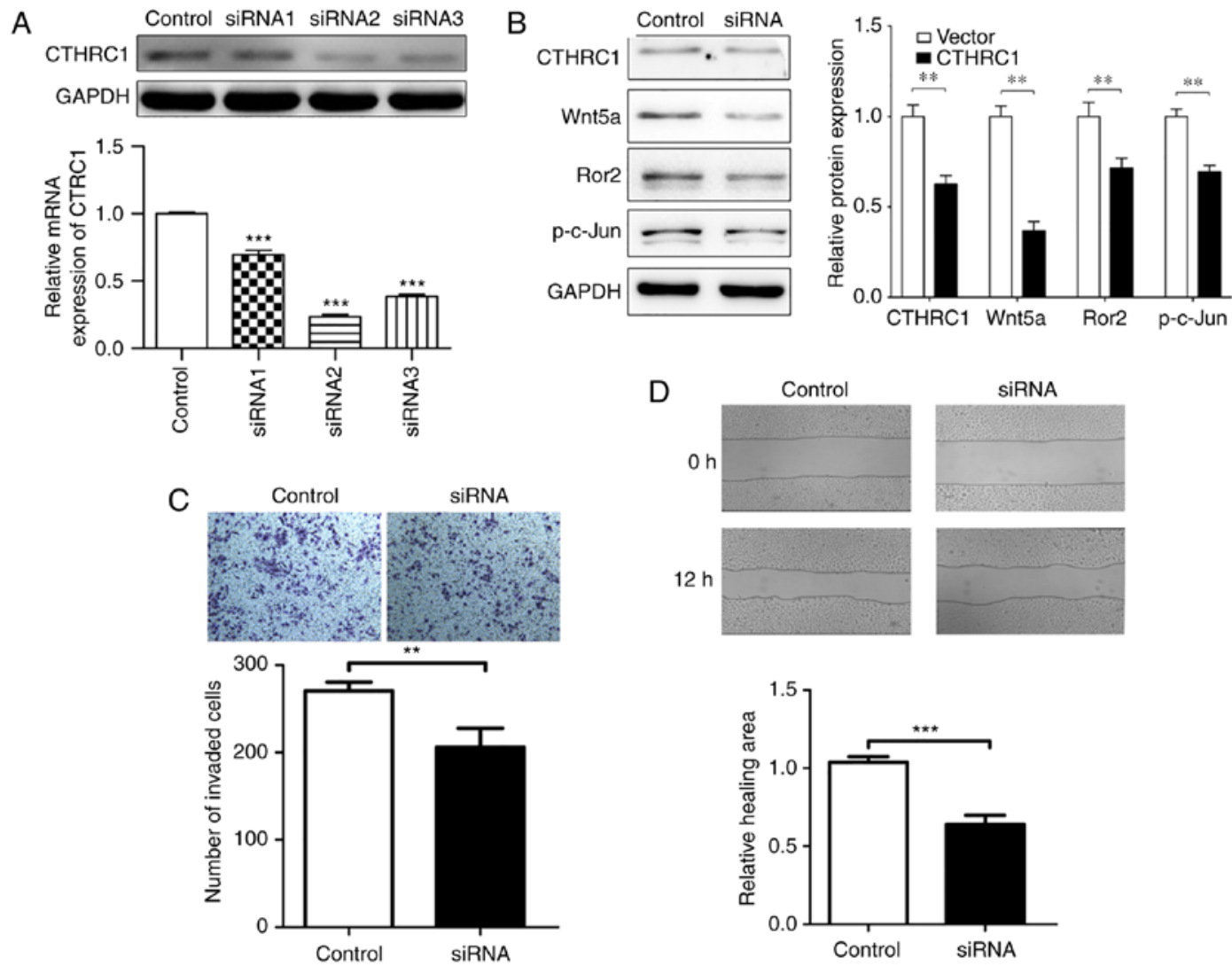

Figure 4. The ability of cell migration and invasion in CTHRC1 knockdown cervical cancer cells. (A) pCDNA3.1 siRNA was chosen the to knock out CTHRC1. (B) The expression of Ror2, Wnt5a and p-c-Jun was downregulated when CTHRC1 was knocked out as revealed by western blotting. (C) Typical images of the Transwell invasion of HeLa cells when CTHRC1 was knocked (scale bar, $100 \mu \mathrm{m}$ ). In each group, 5 stochastic fields were analyzed using Image-Pro Plus 6.0 software. The mean \pm SD was used to count and express the number of invaded cells (CTHRC1 knockout compared to the control, P<0.05). (D) Cell migration was assessed by wound scratch assay after knockout of CTHRC1 (scale bar, $100 \mu \mathrm{m}$ ). Analysis of variance (ANOVA) was used to compare the speed of wound healing between the control and the CTHRC1 group. CTHRC1, collagen triple helix repeat containing $1 .{ }^{* *} \mathrm{P} \leq 0.01 ;{ }^{* * *} \mathrm{P} \leq 0.001$.

A

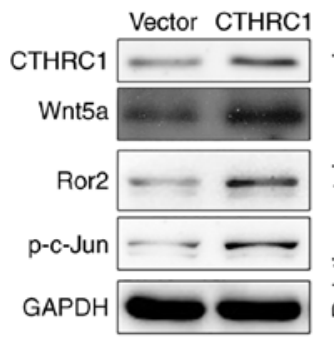

C

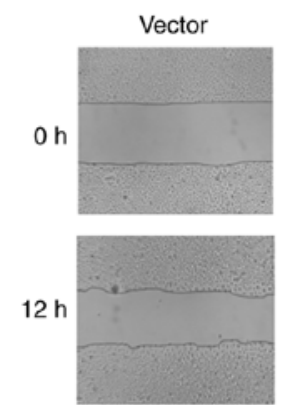

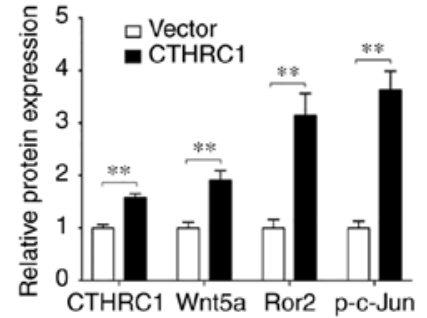

CTHRC1

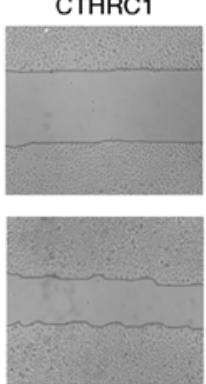

B

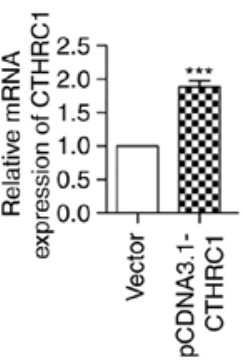

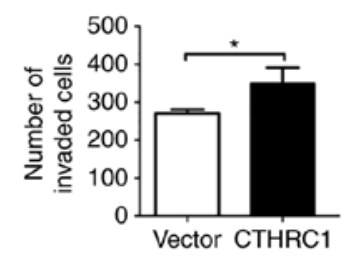

D

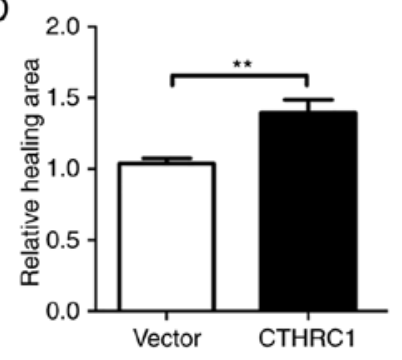

Figure 5. Cell invasion and migration abilities in CTHRC1-overexpressed cervical cancer cells. (A) The expression of p-c-Jun, Ror2 and Wnt5a was increased after overexpression of CTHRC1 as revealed by western blotting. (B) Typical images of the Transwell invasion of HeLa cells when CTHRC1 was overexpressed (scale bar, $100 \mu \mathrm{m}$ ). In each group 5 stochastic fields were analyzed using Image-Pro Plus 6.0 software. The mean \pm SD was used to count and express the number of invaded cells (the group treated with CTHRC1 overexpression was compared with the vector group, $\mathrm{P}<0.05$ ). (C and D) Cell migration was assessed by wound healing assay with overexpressed CTHRC1 (scale bar, $100 \mu \mathrm{m}$ ). Analysis of variance (ANOVA) was used to compare the speed of wound healing between the overexpressed CTHRC1 group and the control group. CTHRC1, collagen triple helix repeat containing $1 .{ }^{*} \mathrm{P} \leq 0.05 ;{ }^{* * *} \mathrm{P} \leq 0.01$. 
indicating that CTHRC1 overexpression influenced the migration behavior of HeLa cells. In Fig. 5B, the number of HeLa cells that invaded to the lower side of the Transwell membrane in the CTHRC1 overexpression group was significantly lower than that in the control group, indicating that CTHRC1 may promote the invasion of HeLa cells. These data demonstrated that CTHRC1 overexpression could regulate and control the invasion and migration of HeLa cells in vitro.

\section{Discussion}

Cervical cancer is one of the most prevalent cancers among women of reproductive age in underdeveloped countries (9). Moreover, the 5-year survival rate of cervical cancer patients is relatively low using traditional treatment. Recurrence and metastasis are the leading causes of death in cervical cancer patients (10). Interactions between extracellular matrix proteins and their receptors initiate downstream signaling pathways leading to tumor invasion and metastasis (11).

CTHRC1 is a $28-\mathrm{kDa}$ extracellular matrix glycoprotein containing an $\mathrm{NH}_{2}$-terminal signaling peptide for extracellular secretion, a short collagen triple-helix repeat of 36 amino acids, and a $\mathrm{COOH}$-terminal globular domain (12). Recent studies have revealed that CTHRC1 is expressed in human cancer and is upregulated in several aggressive tumors, including gastrointestinal, colorectal and breast cancer, and melanoma (13-16). It has been suggested that CTHRC1 protein is undetectable in benign nevi and in non-invasive melanoma tumors, but highly expressed in invasive melanoma. Overexpression of CTHRC1 in melanoma cell lines was revealed to enhance cell migration and adhesion, and protect melanoma cells from serum deprivation-induced apoptosis (17). In breast cancer, the stromal expression of CTHRC1 was enhanced in patients with bone metastasis (18). These studies indicated that CTHRC1 is a critical regulator of tumor development, metastasis and invasion in the tumor microenvironment (19). At present, however, little is known regarding the molecular mechanisms underlying CTHRC1 action in cervical carcinoma. In the present study, we wondered whether CTHRC1 played an important role in promoting migration and invasion of $\mathrm{HeLa}$ cells in vitro. Thus, we revealed the expression of CTHRC1 in cervical carcinoma and investigated the role of CTHRC1 in the invasion and migration of this disease. In our experiment, it was revealed that CTHRC1 was highly expressed in cervical carcinoma and played a role in the invasion and migration of HeLa cells in vitro. It was also inferred that CTHRC1 in HeLa cells markedly promoted the ability of cell invasion and migration when CTHRC1 was overexpressed in vitro as demonstrated by Transwell and wound scratch assays. All of the results indicated that CTHRC1 may be a tumor promoter in human cervical cancer.

The Wnt pathway plays an important role in the microenvironment of cervical cancer carcinogenesis $(20,21)$. The most characterized Wnt canonical pathway determines cell fate and regulation of growth, including the patterning of the neuroectoderm, amplification of neural progenitors and formation of the body axis (22). The non-canonical Wnt/PCP pathway controls cell movement and tissue polarity by activating c-jun N-terminal kinase (JNK), RHOA and nemo-like kinase (NLK) signaling cascades (23). Research suggests that
CTHRC1 can interact with multiple extracellular components of Wnt signaling, including the Wnt/PCP co-receptor Ror2. These components form a CTHRC1/Wnt/Ror2 complex to selectively suppress the Wnt pathway and selectively activate the canonical Wnt/PCP pathway (24) and CTHRC1, as a novel Wnt co-receptor that acts to specifically cluster Wnts with Ror2 and Fzd, leading to activation of the PCP pathway (25). Increasing evidence has suggested that Wnt/Pcp plays an important role in regulating the metastasis of cancer cells (26). In the present study, it was revealed that CTHRC1 could concurrently activate the Wnt/PCP signaling pathway through upregulation of Wnt5a, Ror2 and p-c-Jun proteins to reverse cell migration and invasion. In summary, the Wnt/PCP signal pathway partly explains the mechanism of CTHRC1-induced migration and invasion in HeLa cells in vitro. However, the specific mechanism of this process remains unclear.

In conclusion, our findings demonstrated that CTHRC1 was overexpressed in cervical carcinoma tissues and HeLa cells and played a critical role in cell invasion and migration, which was likely mediated by activation of the Wnt/PCP pathway. Additional experiments with cervical carcinoma are required to demonstrate the specific underlying mechanism of CTHRC1 in cervical cancer progression and metastasis.

\section{Acknowledgements}

Not applicable.

\section{Funding}

The present study was supported by the National Natural Science Foundation of China (no. 81402298), and the Young Scholars Program of Shandong University.

\section{Availability of data and materials}

The datasets used during the present study are available from the corresponding author on reasonable request.

\section{Authors' contributions}

MZ made substantial contributions to the conception and design of the study; QZ made substantial contributions to the analysis and interpretation of the data; XL was involved in the conception of the study, drafted the manuscript and revised it critically for important intellectual content, and was a major contributor in writing the manuscript; $\mathrm{CW}$ performed the histological examination of the cervical carcinoma tissue, and was a major contributor in writing the manuscript; GL contributed to the conception and design of the study and gave final approval of the version to be published and agreement to be accountable for all aspects of the work in ensuring that questions related to the accuracy and integrity of any part of the work are appropriately investigated and resolved. All authors read and approved the final manuscript.

\section{Ethics approval and consent to participate}

All human tissue and sample experiments were approved by the Ethics Committee of the School of Stomatology, 
Shandong University. Informed consent concerning the use of surgically resected specimens for research purposes was obtained from all patients.

\section{Patient consent for publication}

Not applicable.

\section{Competing interests}

The authors state that they have no competing interests.

\section{References}

1. Siegel R, Naishadham D and Jemal A: Cancer statistics, 2013. CA Cancer J Clin 63: 11-30, 2013.

2. Pyagay P, Heroult M, Wang Q, Lehnert W, Belden J, Liaw L, Friesel RE and Lindner V: Collagen triple helix repeat containing 1, a novel secreted protein in injured and diseased arteries, inhibits collagen expression and promotes cell migration. Circ Res 96: 261-268, 2005.

3. Huang CY, Fong YC, Lee CY, Chen MY, Tsai HC, Hsu HC and Tang CH: CCL5 increases lung cancer migration via PI3K, Akt and NF-kappaB pathways. Biochem Pharmacol 77: 794-803, 2009.

4. Kim HC, Kim YS, Oh HW, Kim K, Oh SS, Kim JT, Kim BY,Lee SJ, Choe YK, Kim DH, et al: Collagen triple helix repeat containing 1 (CTHRC1) acts via ERK-dependent induction of MMP9 to promote invasion of colorectal cancer cells. Oncotarget 5: 519-529, 2014.

5. Ma MZ, Zhuang C, Yang XM, Zhang ZZ, Ma H, Zhang WM, You H, Qin W, Gu J, Yang S, et al: CTHRC1 acts as a prognostic factor and promotes invasiveness of gastrointestinal stromal tumors by activating Wnt/PCP-Rho signaling. Neoplasia 16: 265-278, 278.e1-13 2014.

6. Eriksson J, Le Joncour V, Nummela P, Jahkola T, Virolainen S, Laakkonen P, Saksela O and Hölttä E: Gene expression analyses of primary melanomas reveal CTHRC1 as an important player in melanoma progression. Oncotarget 7: 15065-15092, 2016.

7. Puliyappadamba VT, Cheriyan VT, Thulasidasan AK, Bava SV, Vinod BS, Prabhu PR, Varghese R, Bevin A, Venugopal S and Anto RJ: Nicotine-induced survival signaling in lung cancer cells is dependent on their p53 status while its down-regulation by curcumin is independent. Mol Cancer 9: 220, 2010.

8. Maruyama K, Ochiai A, Akimoto S, Nakamura S, Baba S, Moriya Y and Hirohashi S: Cytoplasmic beta-catenin accumulation as a predictor of hematogenous metastasis in human colorectal cancer. Oncology 59: 302-309, 2000.

9. Chidyaonga-Maseko F, Chirwa ML and Muula AS: Underutilization of cervical cancer prevention services in low and middle income countries: A review of contributing factors. Pan Afr Med J 21: 231, 2015.

10. Plummer M, Herrero R, Franceschi S, Meijer CJ, Snijders P, Bosch FX, de Sanjosé S, Muñoz N and IARCMulti-centre Cervical Cancer Study Group: Smoking and cervical cancer: Pooled analysis of the IARC multi-centric case-control study. Cancer Causes Control 14: 805-814, 2003.
11. Multhaupt HA, Leitinger B, Gullberg D and Couchman JR: Extracellular matrix component signaling in cancer. Adv Drug Deliv Rev 97: 28-40, 2016.

12. Hou M, Cheng Z, Shen H, He S, Li Y, Pan Y, Feng C, Chen X, Zhang Y, Lin M, et al: High expression of CTHRC1 promotes EMT of epithelial ovarian cancer (EOC) and is associated with poor prognosis. Oncotarget 6: 35813-35829, 2015.

13. Grando SA: Connections of nicotine to cancer. Nat Rev Cancer 14: 419-429, 2014

14. Tan F, Liu F, Liu H, Hu Y, Liu D, and Li G: CTHRC1 is associated with peritoneal carcinomatosis in colorectal cancer: A new predictor for prognosis. Med Oncol 30: 473, 2013.

15. Tang L, Dai DL, Su M, Martinka M, Li G and Zhou Y: Aberrant expression of collagen triple helix repeat containing 1 in human solid cancers. Clin Cancer Res 12: 3716-3722, 2006.

16. Wang $\mathrm{P}$, Wang YC, Chen XY, Shen ZY, Cao H, Zhang YJ, Yu J, Zhu JD, Lu YY and Fang JY: CTHRC1 is upregulated by promoter demethylation and transforming growth factor- $\beta 1$ and may be associated with metastasis in human gastric cancer. Cancer Sci 103: 1327-1333, 2012.

17. Chen YL, Wang TH, Hsu HC, Yuan RH and Jeng YM: Overexpression of CTHRC1 in hepatocellular carcinoma promotes tumor invasion and predicts poor prognosis. PLoS One 8: e70324, 2013.

18. Kharaishvili G, Cizkova M, Bouchalova K, Mgebrishvili G, Kolar Z and Bouchal J: Collagen triple helix repeat containing 1 protein, periostin and versican in primary and metastatic breast cancer: An immunohistochemical study. J Clin Pathol 64: 977-982, 2011.

19. Lai YH, Chen J, Wang XP, Wu YQ, Peng HT, Lin XH and Wang WJ: Collagen triple helix repeat containing-1 negatively regulated by microRNA-30c promotes cell proliferation and metastasis and indicates poor prognosis in breast cancer. J Exp Clin Cancer Res 36: 92, 2017.

20. Joyce JA and Pollard JW: Microenvironmental regulation of metastasis. Nat Rev Cancer 9: 239-252, 2009.

21. Skeen VR, Paterson I, Paraskeva C and Williams AC: TGF- $\beta 1$ signalling, connecting aberrant inflammation and colorectal tumorigenesis. Curr Pharm Des 18: 3874-3888, 2012.

22. Katoh M: WNT/PCP signaling pathway and human cancer (Review). Oncol Rep 14: 1583-1588, 2005.

23. Logan CY and Nusse R: The Wnt signaling pathway in development and disease. Annu Rev Cell Dev Biol 20: 781-810, 2004.

24. Yamamoto S, Nishimura O, Misaki K, Nishita M, Minami Y, Yonemura S, Tarui $\mathrm{H}$ and Sasaki H: Cthrcl selectively activates the planar cell polarity pathway of Wnt signaling by stabilizing the Wnt-receptor complex. Dev Cell 15: 23-36, 2008.

25. Kelley MW: Leading Wnt down a PCP path: Cthrc1 acts as a coreceptor in the Wnt-PCP pathway. Dev Cell 15: 7-8, 2008.

26. Tsunoda K, Tsujino I, Koshi R, Sugano N, Sato S and Asano M: Nicotine-mediated $\mathrm{Ca}^{2+}$-influx induces IL-8 secretion in oral squamous cell carcinoma cell. J Cell Biochem 117: 1009-1015, 2016.

This work is licensed under a Creative Commons Attribution-NonCommercial-NoDerivatives 4.0 International (CC BY-NC-ND 4.0) License. 